Article

\title{
Qingxuan Jiangya Decoction Reverses Vascular Remodeling by Inducing Vascular Smooth Muscle Cell Apoptosis in Spontaneously Hypertensive Rats
}

\author{
Fei Xiao ${ }^{1,+}{ }^{+}$Fei He ${ }^{2,3,+}$, Hongwei Chen ${ }^{2}$, Shan Lin ${ }^{4}$, Aling Shen ${ }^{2}$, Youqin Chen ${ }^{5}$, \\ Jianfeng $\mathrm{Chu}^{2, *}$ and Jun Peng ${ }^{2, *}$ \\ 1 College of Pharmacy, Fujian University of Traditional Chinese Medicine, 1 Qiuyang Road, Minhou Shangjie, \\ Fuzhou 350122, Fujian, China; ybxwdj@hotmail.com \\ 2 Academy of Integrative Medicine, Fujian University of Traditional Chinese Medicine, 1 Qiuyang Road, \\ Minhou Shangjie, Fuzhou 350122, Fujian, China; hefei1288@163.com (F.H.); 15806018983@163.com (H.C.); \\ saling86@hotmail.com (A.S.) \\ 3 Fuqing Health and Family Planning Bureau, 23 Futang Road, Fuqing 350300, Fujian, China \\ 4 Fujian Key Laboratory of Integrative Medicine on Geriatrics, Fujian University of Traditional Chinese \\ Medicine, 1 Qiuyang Road, Minhou Shangjie, Fuzhou 350122, Fujian, China; lisa3350@163.com \\ 5 Case Western Reserve University School of Medicine, Rainbow Babies and Children's Hospital, \\ Cleveland, OH 44106, USA; yxc571@case.edu \\ * Correspondence: jianfengchu@126.com (J.C.); pjunlab@hotmail.com (J.P.); \\ Tel.: +86-189-5049-8395 (J.C.); +86-591-2286-1303 (J.P.) \\ + These authors contributed equally to this work.
}

Academic Editors: Atanas G. Atanasov, Karel Šmejkal and Elke Heiss Received: 4 May 2016; Accepted: 19 July 2016; Published: 22 July 2016

\begin{abstract}
Qingxuan Jiangya Decoction (QXJYD), a traditional Chinese medicine formula prescribed by academician Ke-ji Chen, has been used in China to clinically treat hypertension for decades of years. However, the molecular mechanisms of its action remain largely unknown. In this study, we examined the therapeutic efficacy of QXJYD against elevated systolic blood pressure in the spontaneously hypertensive rat (SHR) model, and investigated the underlying molecular mechanisms. We found that oral administration of QXJYD significantly reduced the elevation of systolic blood pressure in SHR but had no effect on body weight change. Additionally, QXJYD treatment significantly decreased the media thickness and ratio of media thickness/lumen diameter in the carotid arteries of SHR. Moreover, QXJYD remarkably promoted apoptosis of vascular smooth muscle cells and reduced the expression of anti-apoptotic B-cell leukemia/lymphoma 2. Furthermore, QXJYD significantly decreased the plasma Angiotensin II level in SHR. Collectively, our findings suggest that reversing vascular remodeling via inducing VSMC apoptosis could be one of the mechanisms whereby QXJYD treats hypertension.
\end{abstract}

Keywords: Qingxuan Jiangya Decoction; vascular remodeling; vascular smooth muscle cell; apoptosis; spontaneously hypertensive rats

\section{Introduction}

Hypertension is a key risk factor for various cardiovascular diseases, such as stroke, myocardial infarction, and heart failure [1], accounting for approximately 9.4 million deaths globally each year [2]. It has been predicted that the global prevalence of hypertension will increase by $10 \%$ each year between 2000 and 2025 [3]. Hypertension is a complex and progressive condition which can arise from various genetic and pathogenic causes. Vascular remodeling is an early key outcome of hypertension, characterized by the hypertrophy of vascular smooth muscle cells (VSMCs) and 
increased deposition of extracellular matrix [4]. Although vascular remodeling is initially an adaptive process in response to long-term pressure overload, it contributes to the pathophysiology of vascular diseases, circulatory disorders, and organ damage [4,5]. Clinical and experimental models of hypertension have demonstrated the importance of vascular structure in the regulation of blood pressure [6-8]. Increased vascular mass and arterial wall rigidity accompanied by VSMC hypertrophy or hyperplasia are key contributors to hypertension [9-11]. Vascular remodeling is often characterized by an imbalance between proliferation and apoptosis in VSMCs [12]. Apoptosis plays an essential role during tissue morphogenesis and homeostasis by eliminating damaged or impaired cells, which is tightly regulated by many factors including anti-apoptotic B-cell leukemia/lymphoma 2 (Bcl-2) family members [13,14]. It has been shown that elevated expression of anti-apoptotic Bcl-2 and/or decreased expression of pro-apoptotic Bcl2 associated X protein (Bax) are commonly found in the arteries of spontaneously hypertensive rats (SHR; an effective model for research of hypertension), suggesting that a reduction in apoptosis of damaged VSMCs is involved in the pathogenesis of vascular remodeling [15-17]. Therefore, protection against vascular remodeling via promotion of VSMC apoptosis could be a potential therapeutic target for the treatment of hypertension-associated diseases $[10,11]$.

Traditional Chinese medicines (TCM) have long been used as alternative remedies for a variety of diseases including cardio-cerebrovascular diseases [18-20]. TCM formula Qingxuan Jiangya Decoction (QXJYD), prescribed by academician Ke-ji Chen, has been used in China to clinically treat hypertension for decades of years. In addition, many active components identified in QXJYD, such as rhynchophylline, gastrodin, and baicalin, have been shown to possess anti-hypertension activity [21-24]. However, the molecular mechanisms of QXJYD's action remain largely unknown. Using a SHR model, in the present study we found that QXJYD could significantly reduce elevated systolic blood pressure, demonstrating its therapeutic efficacy against hypertension. To investigate the mechanism mediating the bioactivity of QXJYD, we evaluated its effect on vascular remodeling. Our data showed that QXJYD treatment could significantly decrease the media thickness (MT) and ratio of media thickness/lumen diameter (LD) in the carotid arteries of SHR, indicating that QXJYD can reverse vascular remodeling during hypertension. Moreover, QXJYD remarkably promoted VSMC apoptosis through inhibiting Bcl-2 expression. Therefore, the anti-hypertension activity of QXJYD is probably mediated by reversing vascular remodeling via inducing VSMC apoptosis.

\section{Results}

\subsection{HPLC Fingerprint Analysis of QXJYD}

We prepared and analyzed the chromatographic fingerprint of QXJYD (Figure 1). We estimated gastrodin, rhynchophylline, and baicalin in QXJYD by comparison with each standard compound, which had retention times of $26.42 \mathrm{~min}, 34.45 \mathrm{~min}$, and $36.87 \mathrm{~min}$ (see Supplementary Figure S1).

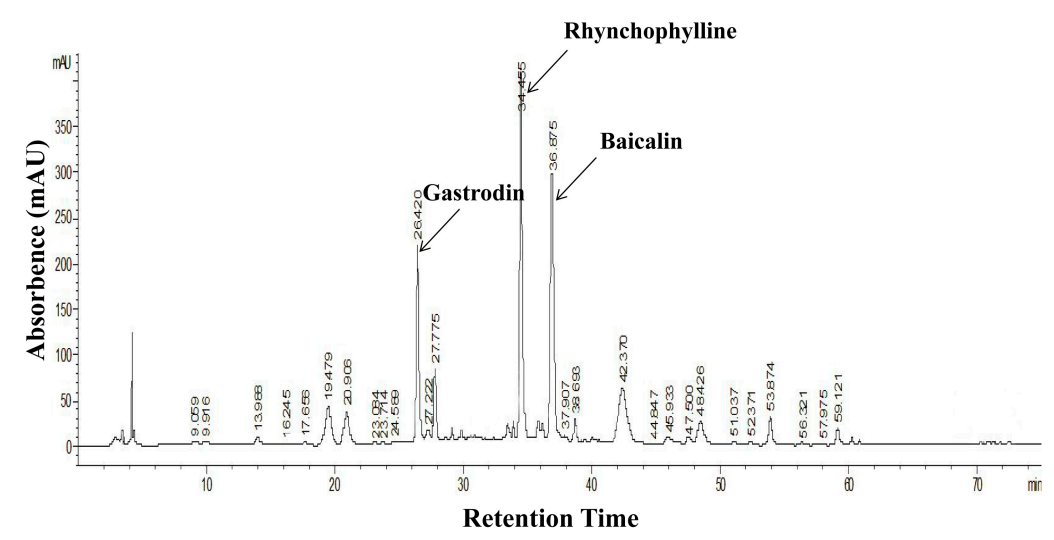

Figure 1. Representative Chromatographic fingerprint of QXJYD. 


\subsection{QXJYD Decreased Elevation of Blood Pressure in SHRs}

The effect of QXJYD on hypertension was evaluated by measurement of systolic blood pressure (SBP) of SHRs, while its adverse effect was examined through determining body weight changes. As as compared with Wistar Kyoto (WKY) rats. However, the elevation of SBP in SHRs was significantly reduced after treatment with QXJYD for five weeks (Figure 2A). At the end of experiment, the average SBP in SHR-control or SHR+QXJYD group was $216 \pm 7$ or $193 \pm 8 \mathrm{mmHg}(n=10)$, respectively. However, administration of QXJYD did not affect body weight change in experimental rats, suggesting no toxicity (Figure 2B).

A

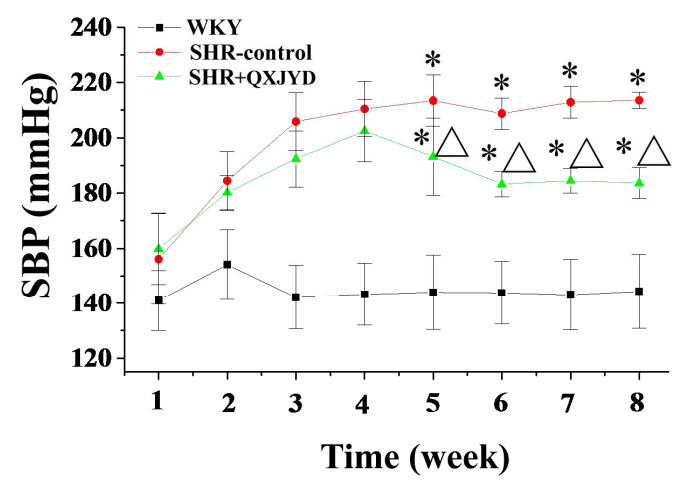

B

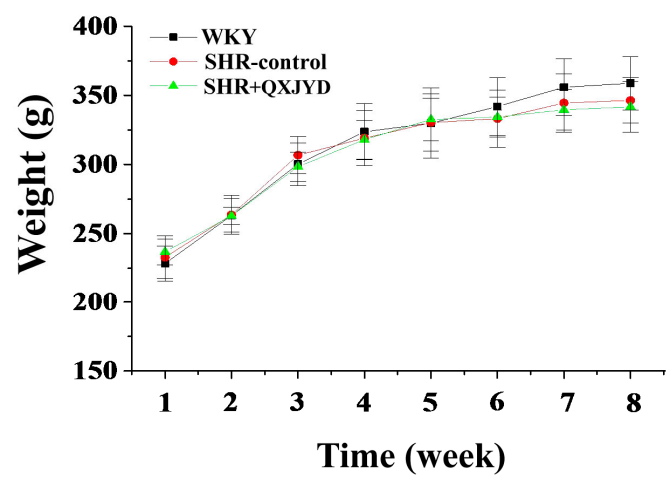

Figure 2. Effect of QXJYD treatment on blood pressure. (A) Systolic blood pressure (SBP); and (B) body weight were measured in spontaneously hypertensive rats (SHR-control, SHR + QXJYD) and Wistar Kyoto (WKY) rats $(n=10)$. All values were represented as mean \pm SD. ${ }^{*} p<0.05$, compared to WKY group; ${ }^{\Delta} p<0.05$, compared to SHR-control group.

\subsection{QXJYD Reversed Vascular Remodeling in SHRs}

The histological changes of thoracic aorta were determined by Harris Hematoxylin and Eosin (H\&E) staining. As shown in Figure 3, although there was no significant difference in LD between the three groups, the MT of thoracic aorta in SHR-control group was significantly greater than that of WKY group, suggesting the vascular remodeling in the rats of the SHR-control group. However, the thickening of thoracic aorta in SHRs was significantly ameliorated by QXJYD treatment. The MT in WKY, SHR-control, or SHR + QXJYD group was $111 \pm 4,165 \pm 4$, or $141 \pm 2 \mu \mathrm{m}$, respectively.

\subsection{QXJYD Promoted VSMC Apoptosis in SHRs}

Apoptosis of aortic VSMCs was determined by terminal deoxynucleotidyl transferase dUTP nick end labeling (TUNEL) assay. As shown in Figure 4, impaired apoptosis might be associated with thickening or vascular remodeling of the thoracic aorta in SHRs, which however was reversed by QXJYD treatment. The percentage of TUNEL-positive cells in WKY, SHR-control, or SHR + QXJYD group was $10.3 \% \pm 2.3 \%, 3.2 \% \pm 1.3 \%$, or $6.3 \% \pm 1.7 \%$, respectively.

\subsection{QXJYD Inhibited Anti-Apoptotic Bcl-2/Bax Ratio in Thoracic Aorta of SHRs}

The protein and mRNA expression levels of Bax and Bcl-2 in VSMCs were assessed by immunohistochemistry and real-time PCR analyses. As shown in Figure 5A, Bcl-2 protein expression in the SHR-control group was significantly increased, as compared to both WKY group. However, hypertension-induced Bcl-2 protein expression was profoundly suppressed by QXJYD treatment. The expression of Bax protein had no difference in all three groups. Data from real-time PCR assay showed that QXJYD significantly reduced Bcl-2 mRNA expression in SHRs, whereas the level of Bax mRNA was significantly increased after QXJYD treatment. Thus, QXJYD-induced apoptosis of VSMCs in hypertentive rats was probably regulated by the inhibition of anti-apoptotic Bcl-2/Bax ratio. 
A

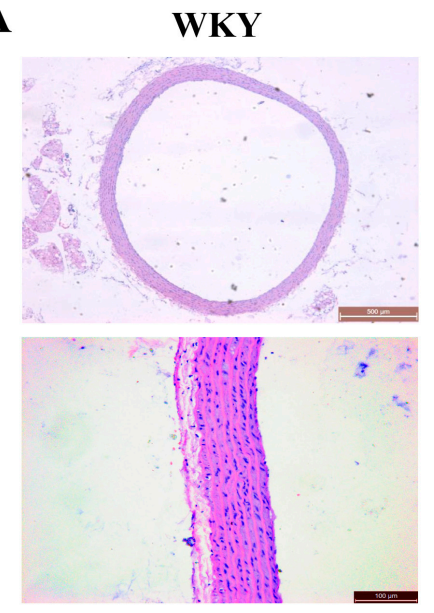

\section{B}

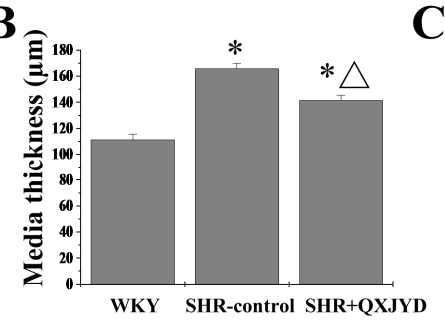

SHR-control

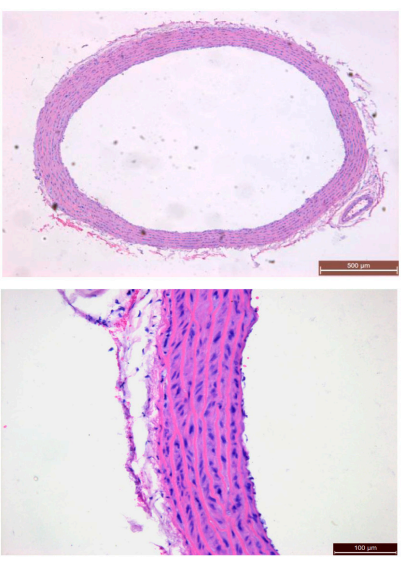

C

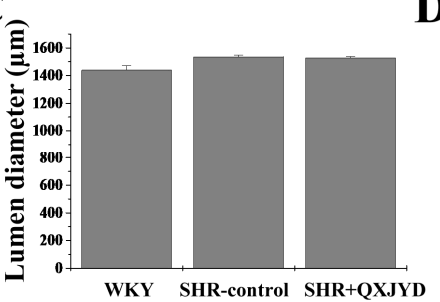

SHR+QXJYD

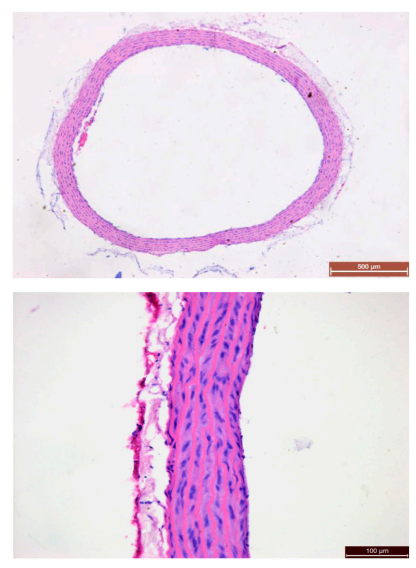

D

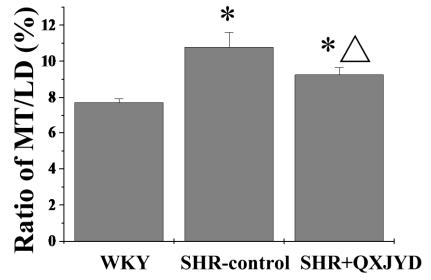

Figure 3. Effect of QXJYD treatment on aortic remodeling. (A) Histopathological changes of thoracic aorta in each group $(n=10)$ was observed by Harris Hematoxylin and Eosin (H\&E) staining. Images were representatives taken at a magnification of $20 \times$ (top, scale bar $=500 \mu \mathrm{m}$ ) or $40 \times$ (bottom, scale bar = $100 \mu \mathrm{m}$ ); (B) Media thickness (MT); (C) Lumen diameter (LD); and (D) MT/LD was measured. All values were represented as mean $\pm \mathrm{SD} ;{ }^{*} p<0.05$ compared to WKY group; ${ }^{\Delta} p<0.05$ compared to SHR-control group.

A

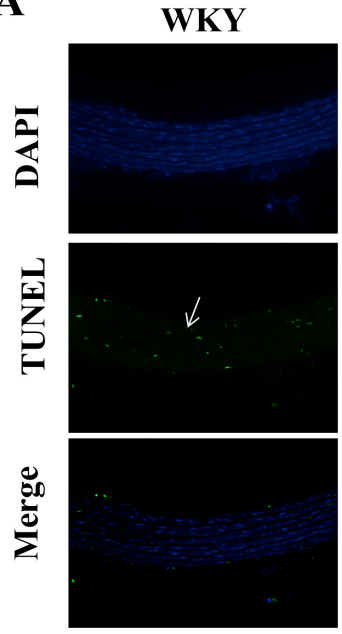

SHR-control

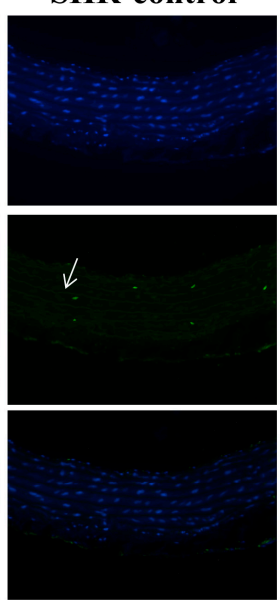

SHR+QXJYD

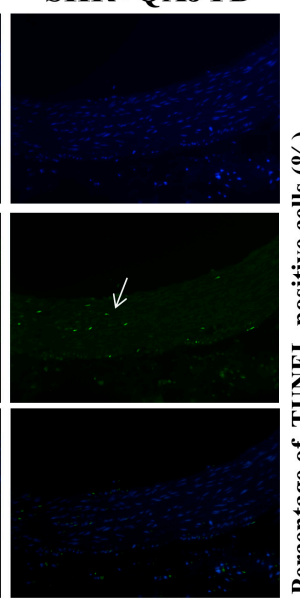

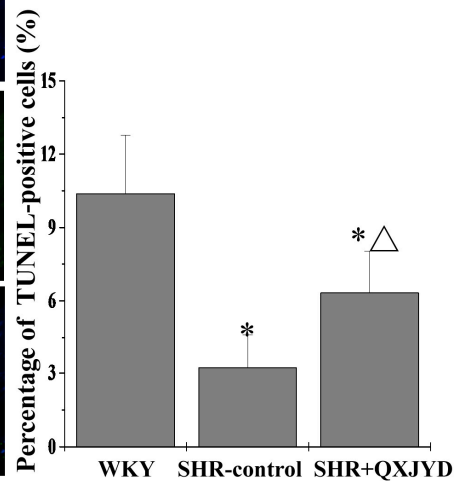

Figure 4. Effect of QXJYD treatment on apoptosis of VSMCs. (A) Thoracic aorta in each group $(n=10)$ was processed for Terminal deoxynucleotidyl transferase dUTP nick end labeling (TUNEL) analysis. Nuclei of all cells were observed through 4,6-diamino-2-phenylindole hydrochloride (DAPI) staining, while apoptotic cells were visualized by green fluorescence. Images were representatives taken by confocal fluorescence microscope at a magnification of 20×; (B) Apoptotic rate was shown as the percentage of TUNEL-positive cells. All values were represented as mean $\pm \mathrm{SD} ;{ }^{*} p<0.05$ compared to WKY group; ${ }^{\Delta} p<0.05$ compared to SHR-control group. 
A

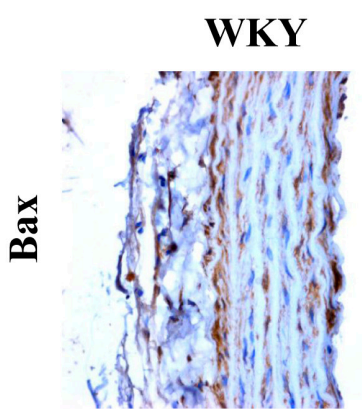

SHR-control
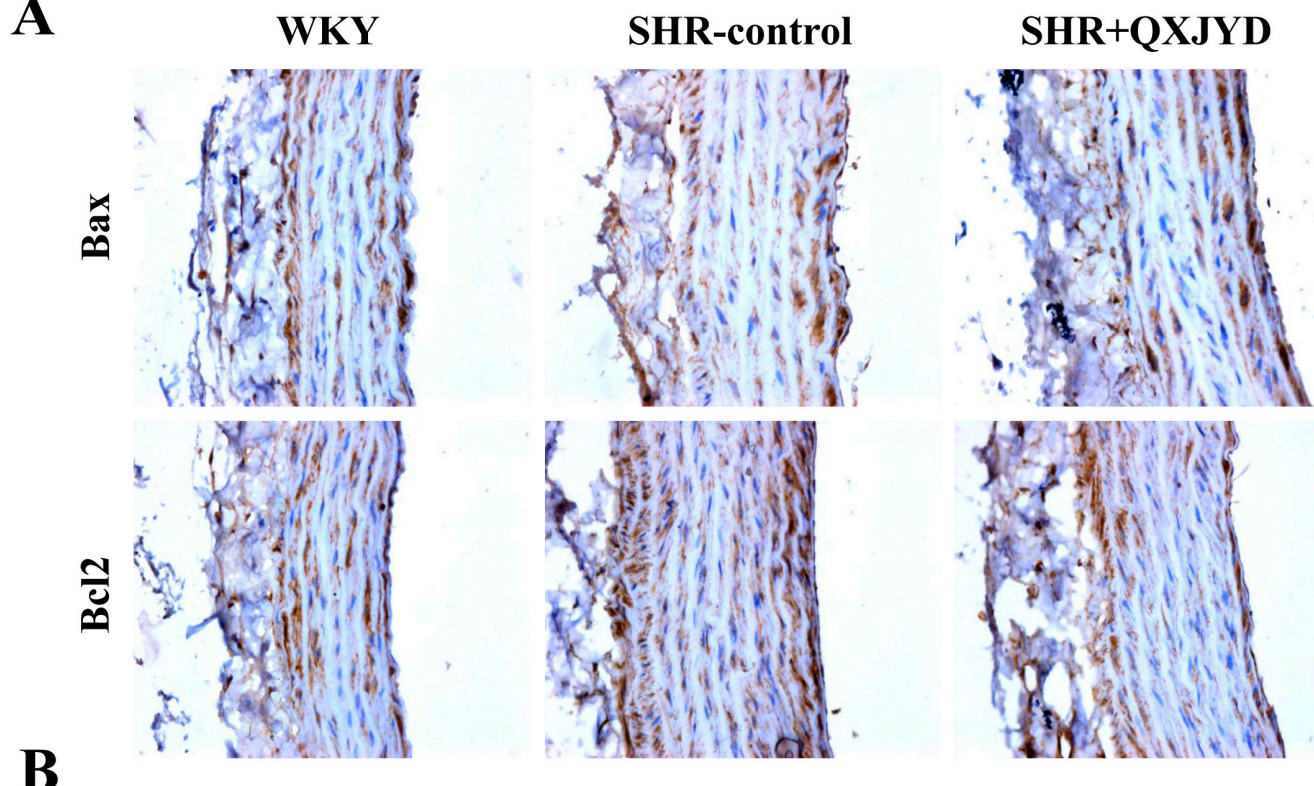

B
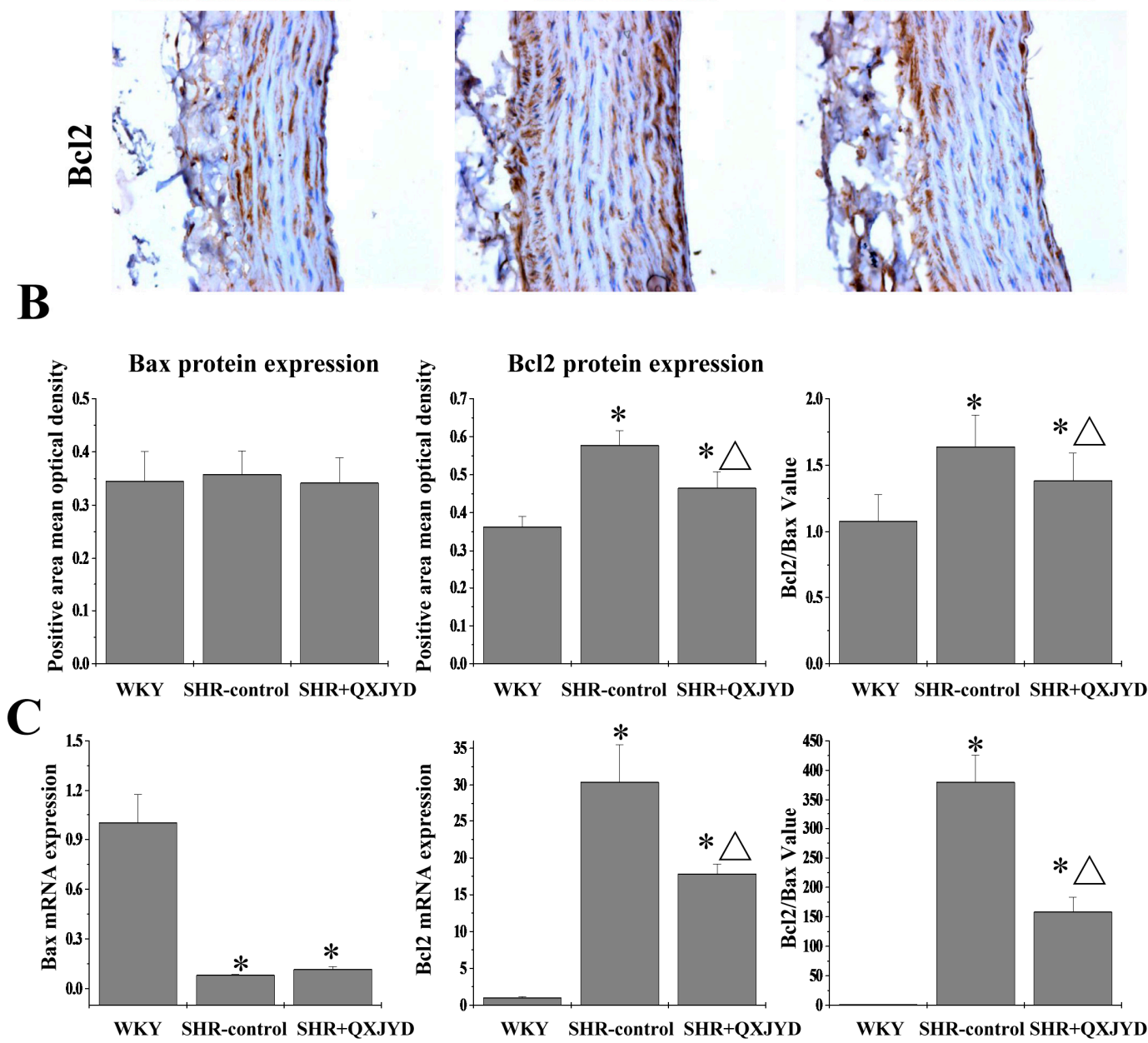

Figure 5. Effect of QXJYD treatment on the expression of Bax and Bcl-2 in VSMCs. (A) Immunohistochemistry analysis was performed to determine the protein expression of Bax and Bcl-2 in thoracic aorta from each group $(n=8)$. Images were representatives taken at a magnification of $20 \times$; (B) Quantification of the mean expressions of Bax and Bcl-2 protein; (C) The mRNA expressions of Bax and Bcl-2 was examined by real-time PCR. All values were represented as mean $\pm \mathrm{SD}$; $p<0.01$ compared to WKY group, ${ }^{\Delta} p<0.05$ compared to SHR-control group.

\subsection{QXJYD Reduced Plasma Ang II Production in SHRs}

Plasma Angiotensin II (Ang II) level was determined by ELISA. As shown in Figure 6, QXJYD treatment could significantly inhibit hypertention-induced Ang II production in vivo. The plasma Ang II level in WKY, SHR-control or SHR + QXJYD group was $0.39 \pm 0.15,1.38 \pm 0.33$, or $0.98 \pm 0.20 \mathrm{ng} / \mathrm{mL}$, respectively. 


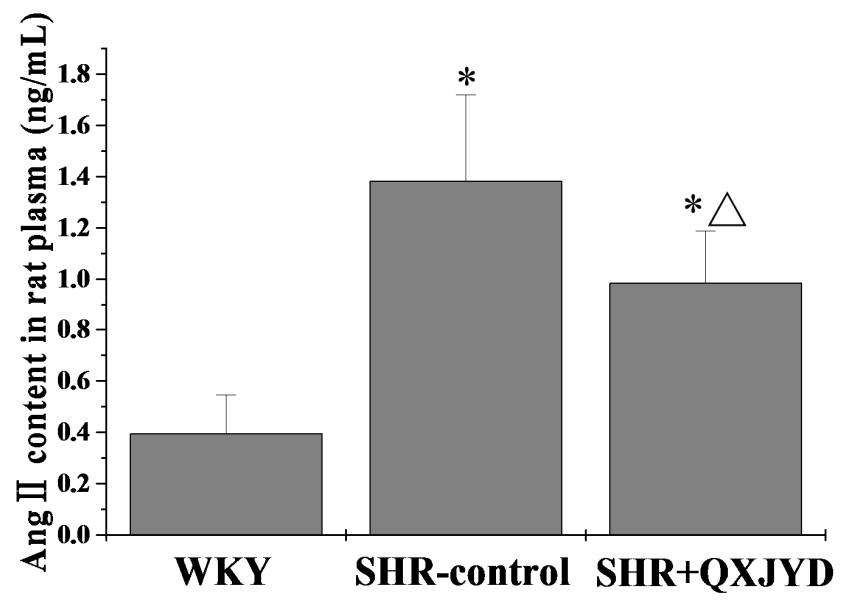

Figure 6. Effect of QXJYD treatment on plasma Ang II production. The level of Angiotensin II (Ang II) in plasma from each group $(n=10)$ was examined by ELISA. All values were represented as mean \pm SD; ${ }^{*} p<0.01$ compared to WKY group, ${ }^{\Delta} p<0.05$ compared to SHR-control group.

\section{Discussion}

Natural products, including TCM, have long been used in China as important alternative remedies for various diseases. As a well-known TCM formula prescribed by Academician Ke-ji Chen, QXJYD has been used in China to clinically treat hypertension for more than 60 years. However, the precise mechanisms of how QXJYD exerts its function remain largely unclear. Therefore, before QXJYD can be further developed as an anti-hypertension agent, the underlying molecular mechanism mediating its bioactivities should first be elucidated.

Using SHR as a hypertension model here, we first demonstrated the therapeutic efficacy of QXJYD against hypertension, as evidenced by its suppressive effect on the increased SBP in SHR model. In addition, QXJYD did not show apparent toxicity since it had no effect on body weight change of experimental animals during the whole course of study. To investigate the mode of action of QXJYD we evaluated its effect on vascular remodeling which is a pathological process associated with progression of hypertension [25-27]. Our data clearly showed that QXJYD treatment could significantly decrease thickening of thoracic aortas in SHR model, indicating its effect of reversing vascular remodeling. The process of vascular remodeling is typically characterized by the hypertrophy of VSMCs, which partially results from the impaired apoptosis. By using TUNEL assay in the present study, we proposed that QXJYD reversed vascular remodeling probably via promotion of apoptosis in aortic VSMCs.

Apoptosis can be triggered by either intrinsic or extrinsic stimuli. The best understood intrinsic apoptotic pathway in vertebrate cells is centered at the mitochondria, which is therefore called mitochondrion-dependent apoptosis. This pathway is tightly regulated by Bcl-2 family members, functioning either as promoter (such as Bax) or as inhibitor (such as Bcl-2). Aberrant expression of $\mathrm{Bcl}-2$ family proteins impairs the normal apoptotic program, resulting in many apoptosis-related diseases. Higher Bcl-2-to-Bax ratios due to the upregulation of Bcl-2 and/or downregulation of Bax expression are commonly found in VSMCs of hypertension. Here we demonstrated that QXJYD treatment suppressed the mRNA expression of Bcl-2 but increased that of Bax. In a consistent manner, QXJYD reduced Bcl-2 protein level although there was no effect on Bax protein expression. Therefore, QXJYD induced apoptosis by decreasing anti-apoptotic Bcl-2/Bax ratio in VSMCs of SHR model.

Ang II is a well-known regulator of VSMC hypertrophy. Ang II has been reported to act as a bifunctional modulator of VSMC apoptosis through either the anti-apoptotic, angiotensin type 1 receptor (AT1R), or the pro-apoptotic angiotensin type 2 receptor (AT2R) [28]. Previous studies have shown that Ang II can induce apoptosis in AT2R transfected VSMCs and protect native VSMCs against apoptosis via AT1Rs [29]. During vascular remodeling, VSMC often shows excessive proliferative and 
hypertrophic activities [30] through the increase in phosphorylation of extracellular signal regulated kinase 1/2 [31]. In order to evaluate Angiotensin-converting enzyme (ACE)—specifically cleaving Ang I into Ang II-inhibitory activity of QXJYD, we measured Ang II content in rats' plasma. Results from this study indicated that QXJYD treatment significantly reduced plasma Ang II production in SHR model, which was consistent with the inhibitory effect of QXJYD on vascular remodeling and VSMC apoptosis. Components of QXJYD may inhibit ACE in vivo. Collectively, our findings suggest that reversing vascular remodeling via inducing VSMC apoptosis could be one of the mechanisms whereby QXJYD treats hypertension.

Besides Ang II, there are many other mechanisms involved in the balance of apoptosis and proliferation of VSMCs, such as NF-E2-related factor 2 (Nrf2)/Kelch-like ECH-associated protein 1 (Keap1) system [32], Phosphatase and Tensin homolog deleted on chromosome 10 (PTEN)/Protein Kinase B (AKT) pathway [33] and Ras homolog gene family, member A (RhoA)/Rho-associated protein kinase (ROCK) pathways [34]. In addition, like other TCM formulas, QXJYD is a combination of several natural products, all of which contain numerous chemical compounds including rhynchophylline, gastrodin, and baicalin [21-24]. It is unknown how many of these compounds contain anti-hypertensive activity. The signaling pathway(s) with which these components exert their function remains unknown also. These intriguing questions should be clarified by future studies before we fully explore the molecular mechanisms of therapeutic effects of QXJYD and develop better drugs for treatment of hypertension.

\section{Materials and Methods}

\subsection{Materials and Reagents}

TUNEL FITC apoptosis detection kit was purchased from Vazyme Biotech Co., Ltd. (Nanjing, China). Histostain-Plus Kit was purchased from Mai Xin biotechnology (Fuzhou, China). Bax, Bcl-2, and $\beta$-actin primers were synthesized by Invitrogen (Grand Island, NY, USA). TRIzol reagent, PrimeScript ${ }^{\mathrm{TM}}$ RT reagent Kit and SYBR Premix Ex Taq II Kit were provided by Takara Biotechnology Co., Ltd. (Dalian, Liaoning, China). Bcl-2, Bax antibodies and horseradish peroxidase (HRP)-conjugated secondary antibodies were obtained from Cell Signaling Technology (Beverly, MA, USA). Ang II rat ELISA kit was purchased from Westang Biotech Co., Ltd. (Shanghai, China). All other chemicals were obtained from Sigma Chemicals (St. Louis, MO, USA) unless otherwise stated.

\subsection{Preparation and HPLC Analysis of QXJYD}

$210 \mathrm{~g}$ of QXJYD obtained from the Third Affiliated Hospital of Fujian University of Traditional Chinese Medicine was extracted with $1000 \mathrm{~mL}$ of distilled water using reflux method and was filtered twice. The water solvent was evaporated on a rotary evaporator (Yarong, Model RE-2000, Shanghai, China). The resulting solution was concentrated to produce $28.91 \mathrm{~g}$ dried water extract of QXJYD. $28 \mathrm{~g}$ of dried water extract was then re-suspended in $70 \mathrm{~mL}$ of $0.9 \% \mathrm{NaCl}$ solution and stored at $4{ }^{\circ} \mathrm{C}$ for subsequent animal experiments. The remaining dried QXJYD was dissolved in $1 \mathrm{~mL}$ absolute methanol. In order to improve the solubility of dried QXJYD, sonication was performed for $30 \mathrm{~min}$. This solution was subsequently partitioned using a separating funnel and stored at $4{ }^{\circ} \mathrm{C}$ for principal component analysis using High Performance Liquid Chromatography (HPLC).

HPLC analysis was carried using an Aglient 1200 HPLC system (Cold Spring, NY, USA). QXJYD was diluted to a final concentration of $50 \mathrm{mg} / \mathrm{mL}$ in methanol. Rhynchophylline, gastrodin, and baicalin standards were used to identify the components in QXJYD. The analysis was performed on phenomenex luna C18 reverse phase column $(250 \mathrm{~mm} \times 4.6 \mathrm{~mm}$ I.D., $5 \mu \mathrm{m})$. The flow rate was $1 \mathrm{~mL} / \mathrm{min}$ and injection volume was $10 \mu \mathrm{L}$. The detection wavelength was $280.8 \mathrm{~nm}$ and oven temperature was set to $37^{\circ} \mathrm{C}$. The mobile phase consisted of $0.1 \%$ phosphoric acid aqueous solution (eluent A) and methanol (eluent B) with the following gradient program: $5 \%$ eluent B from 0 to 10 min; $10 \%-40 \%$ eluent B from 10 to $30 \mathrm{~min}$, and 40\%-100\% eluent B from 30 to $75 \mathrm{~min}$. 


\subsection{Animals}

Twenty male SHRs (age: 7 weeks; weight: $200 \pm 20 \mathrm{~g}$ ) and 10 male WKYs (age: 7 weeks; weight: $200 \pm 20 \mathrm{~g}$ ) were obtained from Slac Laboratory Animal Technology Co., Ltd. (Shanghai, China). All rats were housed in clean specific pathogen-free rooms with controlled temperature $\left(22{ }^{\circ} \mathrm{C}\right)$, humidity, and a 12-h light/dark cycle. Food and water were provided ad libitum throughout the experiment. All animal experiments were performed strictly in accordance with international ethical guidelines and the National Institutes of Health Guide concerning the care and use of laboratory animals, and were approved by the Institutional Animal Care and Use Committee of Fujian University of Traditional Chinese Medicine.

\subsection{Drug Administration and Blood Pressure Measurement}

After acclimation for one week, rats were divided into three groups $(n=10)$, termed as WKY, SHR-control and SHR + QXJYD. Rats in SHR + QXJYD group were orally treated with $60 \mathrm{mg} / \mathrm{kg}$ of QXJYD every day, while rats in WKY and SHR-control group were treated with saline solution. Systolic blood pressures of all rats were measured once per week, with the tail-cuff plethysmograph method using CODA ${ }^{\mathrm{TM}}$ non-invasive blood pressure system (Kent Scientific, Torrington, CT, USA).

\subsection{Vessel Morphometry}

Artery morphological characteristics were assessed using H\&E Staining. Paraffin-embedded $5 \mu \mathrm{m}$-thick sections of aorta samples were stained with Harris Hematoxylin for 2 min then washed under running tap water $5 \mathrm{~min}$. Slides were then differentiated in $1 \%$ acid alcohol $30 \mathrm{~s}$, then washed under running tap water $5 \mathrm{~min}$, before staining with Eosin for $1 \mathrm{~min}$. Following gradient dehydration and drying, samples were measured using true color multi-functional cell image analysis system (Image-Pro Plus, Media Cybernetics, Rockville, MD, USA). The aortic media was defined as the region between the internal and external elastic laminae; the intima area was defined as the region between the endothelium and the internal elastic laminae; the lumen area was defined as the area encompassed by the endothelium. The morphometric measurements for each vessel were calculated based on the average of its proximal and distal regions. VSMCs were stained blue in the tunica media.

\subsection{In Situ Apoptosis Detection by TUNEL Staining}

$5 \mu \mathrm{m}$-thick sections of aorta samples were analyzed using TUNEL fluorescein isothiocyanate (FITC) apoptosis detection kit (Vazyme Biotech Co., Ltd.). TUNEL-positive cells exhibited apoptosis characteristics including condensed chromatin and cellular shrinkage. Apoptotic cells were counted as FITC-positive cells (stained green) and total number of cells was counted as 4,6-diamino-2-phenylindole hydrochloride (DAPI) (stained blue) in five randomly selected fields for each slide. The percentage of apoptotic cells was calculated as the ratio of the number of TUNEL-positive cells to the total number of cells, as the mean of the five randomly selected fields. The sections of aorta samples after deparaffinage were washed with PBS and incubated for $10 \mathrm{~min}$ with DAPI protected from light. The cover slips were sealed onto slides with nail polish and stored at $-20^{\circ} \mathrm{C}$ until image collection by florescence microscopy.

\subsection{Immunohistochemistry Analysis}

Bax and Bcl-2 expression were assessed by immunohistochemistry. Briefly, $5 \mu \mathrm{m}$ cross sections of arteries were fixed in $4 \%$ paraformaldehyde $(\mathrm{pH}=7.4)$. The slides were subjected to antigen retrieval and the endogenous peroxidase activity was blocked with 3\% hydrogen peroxide. Slides were incubated with rabbit polyclonal antibodies against Bax (1:200) and Bcl-2 (1:200) (Cell Signaling Technology). After washing with PBS, slides were incubated with biotinylated secondary antibody followed by conjugated HRP-labelled strepta-vidin (Maixing), then washed with PBS. The slides were then incubated with diaminobenzidine (Maixing) as the chromogen, followed by counterstaining with 
diluted Harris hematoxylin (Maixing). Five microscopic fields (magnification $400 \times$ ) were randomly selected in each slide, and the average optical density of positive cells in each field were measured using true color multi-functional cell image analysis system (Image-Pro Plus, Media Cybernetics). To rule out any non-specific staining, PBS was used instead of primary antibody as the negative control.

\subsection{RNA Extraction and Quantitative PCR Analysis}

Total RNA was extracted using TRIzol reagent (Takara Biotechnology Co., Ltd.) according to the manufacturer's instructions. To synthesize cDNA, $1 \mu \mathrm{g}$ total RNA was reverse-transcribed using a Prime Script ${ }^{\circledR}$ II 1st strand cDNA Synthesis kit (Takara Biotechnology Co., Ltd.). Real-time fluorescence quantitative PCR was performed using SYBR-Green premix (Applied Biosystems, Carlsbad, CA, USA) according to the manufacturer's instructions, using the following parameters: 40 cycles; $50{ }^{\circ} \mathrm{C}$ for $2 \mathrm{~min}, 95^{\circ} \mathrm{C}$ for $7 \mathrm{~min}, 60^{\circ} \mathrm{C}$ for $15 \mathrm{~s}$, and $60{ }^{\circ} \mathrm{C}$ for $30 \mathrm{~s}$. $\beta$-actin was used as the internal control. PCR primer sequences $\left(5^{\prime} \rightarrow 3^{\prime}\right)$ as shown in the Table 1 :

Table 1. The primer sequences for quantitative PCR analysis.

\begin{tabular}{cl}
\hline Gene Name & \multicolumn{1}{c}{ Sequence } \\
\hline \multirow{2}{*}{$\beta$-actin } & F: 5'-TGTCACCAACTGGGACGATA-3' \\
& R: 5'-GGGGTGTTGAAGGTCTCAAA-3' \\
\hline \multirow{2}{*}{ Bax } & F: 5'-TGCTACAGGGTTTCATCCAG-3' \\
& R: 5'-TGTTGTTGTCCAGTTCATCG-3' \\
\hline \multirow{2}{*}{ Bcl-2 } & F: 5'-GGTGGACAACATCGCTCTG-3' \\
& R: 5'-ACAGCCAGGAGAAATCAAACA-3' \\
\hline
\end{tabular}

\subsection{Measurement of Ang II in Rat Plasma}

The blood samples collected during exsanguination were transferred to a tube containing $10 \%$ EDTA-2K anticoagulant. The uncoagulated blood was centrifuged at $2000 \times g$ for $10 \mathrm{~min}$ at $4{ }^{\circ} \mathrm{C}$ to separate the plasma. Measurement of the total Ang II in the plasma was performed using an ELISA kit according to the manufacturer's instructions.

\subsection{Statistical Analysis}

All data were expressed as mean \pm standard deviation (SD). Statistical significance was calculated with one-way analysis of variance using SPSS software (version 18.0; SPSS, Inc., Chicago, IL, USA). $p<0.05$ was considered as statistically significant.

\section{Conclusions}

In the present study, we demonstrated that the TCM formula QXJYD could significantly reduce the elevation of systolic blood pressure in an SHR model but had no effect on body weight changes, indicating its therapeutic efficacy against hypertension without apparent toxicity. QXJYD treatment significantly decreased the thickening of thoracic aorta in SHR model, suggesting its activity of reversing vascular remodeling during hypertension. QXJYD remarkably promoted apoptosis of VSMCs and reduced the expression of anti-apoptotic Bcl-2. Taken together, it is suggested that reversing vascular remodeling via promoting VSMC apoptosis might be one of the mechanisms by which QXJYD exerts its anti-hypertension effects.

Supplementary Materials: Supplementary materials can be accessed at: http://www.mdpi.com/1420-3049/ 21/7/956/s1.

Acknowledgments: This study was sponsored by the National Natural Science Foundations of China (81302884), the China Postdoctoral Science Foundation (2013T60636), and the Developmental Fund of Chen Keji Integrative Medicine (CKJ2013009). 
Author Contributions: F.X., J.F., Y.Q., and J.P. conceived and designed the experiments; F.X. and F.H. performed the experiments; H.W. and S.L. analyzed the data; A.S. contributed reagents/materials/analysis tools; F.X., J.F., and J.P. wrote the paper.

Conflicts of Interest: The authors declare no conflict of interest.

\section{References}

1. Piper, M.A.; Evans, C.V.; Burda, B.U.; Margolis, K.L.; O'Connor, E.; Smith, N.; Webber, E.; Perdue, L.A.; Bigler, K.D.; Whitlock, E.P. U.S. Preventive Services Task Force Evidence Syntheses, formerly Systematic Evidence Reviews. In Screening for High Blood Pressure in Adults: A Systematic Evidence Review for the U.S. Preventive Services Task Force; Agency for Healthcare Research and Quality: Rockville, MD, USA, 2014.

2. Lim, S.S.; Vos, T.; Flaxman, A.D.; Danaei, G.; Shibuya, K.; Adair-Rohani, H.; Amann, M.; Anderson, H.R.; Andrews, K.G.; Aryee, M.; et al. A comparative risk assessment of burden of disease and injury attributable to 67 risk factors and risk factor clusters in 21 regions, 1990-2010: A systematic analysis for the global burden of disease study 2010. Lancet 2012, 380, 2224-2260. [CrossRef]

3. Poulter, N.R.; Prabhakaran, D.; Caulfield, M. Hypertension. Lancet 2015, 386, 801-812. [CrossRef]

4. Drummond, G.R.; Selemidis, S.; Griendling, K.K.; Sobey, C.G. Combating oxidative stress in vascular disease: Nadph oxidases as therapeutic targets. Nat. Rev. Drug Discov. 2011, 10, 453-471. [CrossRef] [PubMed]

5. Birns, J.; Markus, H.; Kalra, L. Blood pressure reduction for vascular risk: Is there a price to be paid? Stroke J. Cereb. Circ. 2005, 36, 1308-1313. [CrossRef] [PubMed]

6. Rizzoni, D.; Agabiti-Rosei, E. Structural abnormalities of small resistance arteries in essential hypertension. Intern. Emerg. Med. 2012, 7, 205-212. [CrossRef] [PubMed]

7. Heistad, D.D.; Armstrong, M.L.; Baumbach, G.L.; Faraci, F.M. Sick vessel syndrome. Recovery of atherosclerotic and hypertensive vessels. Hypertension 1995, 26, 509-513. [CrossRef] [PubMed]

8. Folkow, B. Hypertensive structural changes in systemic precapillary resistance vessels: How important are they for in vivo haemodynamics? J. Hypertens. 1995, 13, 1546-1559. [CrossRef] [PubMed]

9. Boutouyrie, P.; Bussy, C.; Lacolley, P.; Girerd, X.; Laloux, B.; Laurent, S. Association between local pulse pressure, mean blood pressure, and large-artery remodeling. Circulation 1999, 100, 1387-1393. [CrossRef] [PubMed]

10. Marchand, E.L.; Der Sarkissian, S.; Hamet, P.; deBlois, D. Caspase-dependent cell death mediates the early phase of aortic hypertrophy regression in losartan-treated spontaneously hypertensive rats. Circ. Res. 2003, 92, 777-784. [CrossRef] [PubMed]

11. Baker, A.B.; Ettenson, D.S.; Jonas, M.; Nugent, M.A.; Iozzo, R.V.; Edelman, E.R. Endothelial cells provide feedback control for vascular remodeling through a mechanosensitive autocrine TGF-beta signaling pathway. Circ. Res. 2008, 103, 289-297. [CrossRef] [PubMed]

12. McMurtry, M.S.; Bonnet, S.; Wu, X.; Dyck, J.R.; Haromy, A.; Hashimoto, K.; Michelakis, E.D. Dichloroacetate prevents and reverses pulmonary hypertension by inducing pulmonary artery smooth muscle cell apoptosis. Circ. Res. 2004, 95, 830-840. [CrossRef] [PubMed]

13. Kerr, J.F.; Wyllie, A.H.; Currie, A.R. Apoptosis: A basic biological phenomenon with wide-ranging implications in tissue kinetics. Br. J. Cancer 1972, 26, 239-257. [CrossRef] [PubMed]

14. Thompson, C.B. Apoptosis in the pathogenesis and treatment of disease. Science 1995, 267, $1456-1462$. [CrossRef] [PubMed]

15. Vega, F.; Panizo, A.; Pardo-Mindan, J.; Diez, J. Susceptibility to apoptosis measured by MYC, BCL-2, and BAX expression in arterioles and capillaries of adult spontaneously hypertensive rats. Am. J. Hypertens. 1999, 12, 815-820. [CrossRef]

16. Bennett, M.R.; Evan, G.I.; Newby, A.C. Deregulated expression of the c-MYC oncogene abolishes inhibition of proliferation of rat vascular smooth muscle cells by serum reduction, interferon-gamma, heparin, and cyclic nucleotide analogues and induces apoptosis. Circ. Res. 1994, 74, 525-536. [CrossRef] [PubMed]

17. Diez, J.; Panizo, A.; Hernandez, M.; Pardo, J. Is the regulation of apoptosis altered in smooth muscle cells of adult spontaneously hypertensive rats? Hypertension 1997, 29, 776-780. [CrossRef] [PubMed]

18. Zhang, Y.; Yu, T.; Xu, J.; Bian, X.; Xu, J.; Liu, Y. Effect and mechanism of gastrodin in relaxing isolated thoracic aorta rings in rats. Zhongguo Zhong Yao Za Zhi 2012, 37, 2135-2138. [PubMed] 
19. Kim, E.Y.; Rhyu, M.R. Synergistic vasorelaxant and antihypertensive effects of Ligusticum wallichii and Angelica gigas. J. Ethnopharmacol. 2010, 130, 545-551. [CrossRef] [PubMed]

20. Huang, H.; Zhong, R.; Xia, Z.; Song, J.; Feng, L. Neuroprotective effects of rhynchophylline against ischemic brain injury via regulation of the AKT/mtor and tlrs signaling pathways. Molecules 2014, 19, 11196. [CrossRef] [PubMed]

21. Guo, H.; Zhang, X.; Cui, Y.; Deng, W.; Xu, D.; Han, H.; Wang, H.; Chen, Y.; Li, Y.; Wu, D. Isorhynchophylline protects against pulmonary arterial hypertension and suppresses pasmcs proliferation. Biochem. Biophys. Res. Commun. 2014, 450, 729-734. [CrossRef] [PubMed]

22. Tsai, D.S.; Chang, Y.S.; Li, T.C.; Peng, W.H. Prescription pattern of Chinese herbal products for hypertension in Taiwan: A population-based study. J. Ethnopharmacol. 2014, 155, 1534-1540. [CrossRef] [PubMed]

23. Luan, Y.; Chao, S.; Ju, Z.Y.; Wang, J.; Xue, X.; Qi, T.G.; Cheng, G.H.; Kong, F. Therapeutic effects of baicalin on monocrotaline-induced pulmonary arterial hypertension by inhibiting inflammatory response. Int. Immunopharmacol. 2015, 26, 188-193. [CrossRef] [PubMed]

24. Liu, P.; Yan, S.; Chen, M.; Chen, A.; Yao, D.; Xu, X.; Cai, X.; Wang, L.; Huang, X. Effects of baicalin on collagen iota and collagen iotaiotaiota expression in pulmonary arteries of rats with hypoxic pulmonary hypertension. Int. J. Mol. Med. 2015, 35, 901-908. [PubMed]

25. Hillege, H.L.; Girbes, A.R.; de Kam, P.J.; Boomsma, F.; de Zeeuw, D.; Charlesworth, A.; Hampton, J.R.; van Veldhuisen, D.J. Renal function, neurohormonal activation, and survival in patients with chronic heart failure. Circulation 2000, 102, 203-210. [CrossRef] [PubMed]

26. Laurent, S.; Boutouyrie, P. The structural factor of hypertension: Large and small artery alterations. Circ. Res. 2015, 116, 1007-1021. [CrossRef] [PubMed]

27. Hosoo, S.; Koyama, M.; Kato, M.; Hirata, T.; Yamaguchi, Y.; Yamasaki, H.; Wada, A.; Wada, K.; Nishibe, S.; Nakamura, K. The restorative effects of Eucommia ulmoides oliver leaf extract on vascular function in spontaneously hypertensive rats. Molecules 2015, 20, 21971-21981. [CrossRef] [PubMed]

28. Bascands, J.L.; Girolami, J.P.; Troly, M.; Escargueil-Blanc, I.; Nazzal, D.; Salvayre, R.; Blaes, N. Angiotensin II induces phenotype-dependent apoptosis in vascular smooth muscle cells. Hypertension 2001, 38, 1294-1299. [CrossRef] [PubMed]

29. Yamada, T.; Akishita, M.; Pollman, M.J.; Gibbons, G.H.; Dzau, V.J.; Horiuchi, M. Angiotensin II type 2 receptor mediates vascular smooth muscle cell apoptosis and antagonizes angiotensin II type 1 receptor action: An in vitro gene transfer study. Life Sci. 1998, 63, PL289-PL295. [CrossRef]

30. Fang, H.; Chen, W.; Gao, Y.; Shen, Y.; Luo, M. Molecular mechanisms associated with angiotensin-converting enzyme-inhibitory peptide activity on vascular extracellular matrix remodeling. Cardiology 2014, 127, 247-255. [CrossRef] [PubMed]

31. Lovdahl, C.; Thyberg, J.; Hultgardh-Nilsson, A. The synthetic metalloproteinase inhibitor batimastat suppresses injury-induced phosphorylation of map kinase ERK1/ERK2 and phenotypic modification of arterial smooth muscle cells in vitro. J. Vasc. Res. 2000, 37, 345-354. [CrossRef] [PubMed]

32. Ashino, T.; Yamamoto, M.; Numazawa, S. NRF2/keap1 system regulates vascular smooth muscle cell apoptosis for vascular homeostasis: Role in neointimal formation after vascular injury. Sci. Rep. 2016, 6, 26291. [CrossRef] [PubMed]

33. Liu, Y.; Cao, Y.; Sun, S.; Zhu, J.; Gao, S.; Pang, J.; Zhu, D.; Sun, Z. Transforming growth factor-beta1 upregulation triggers pulmonary artery smooth muscle cell proliferation and apoptosis imbalance in rats with hypoxic pulmonary hypertension via the PTEN/AKT pathways. Int. J. Biochem. Cell Biol. 2016, 77, 141-154. [CrossRef] [PubMed]

34. Nour-Eldine, W.; Ghantous, C.M.; Zibara, K.; Dib, L.; Issaa, H.; Itani, H.A.; El-Zein, N.; Zeidan, A. Adiponectin attenuates angiotensin II-induced vascular smooth muscle cell remodeling through nitric oxide and the rhoa/rock pathway. Front. Pharmacol. 2016, 7, 86. [CrossRef] [PubMed]

Sample Availability: Not available.

(C) 2016 by the authors; licensee MDPI, Basel, Switzerland. This article is an open access article distributed under the terms and conditions of the Creative Commons Attribution (CC-BY) license (http://creativecommons.org/licenses/by/4.0/). 